\title{
Towards Mobile 3D Telepresence Using Head-worn Devices and Dual- Purpose Screens
}

\author{
Shoaib R. Soomro* \\ Koç University
}

\author{
Osman Eldes
}

Koç University
Hakan Urey

Koç University

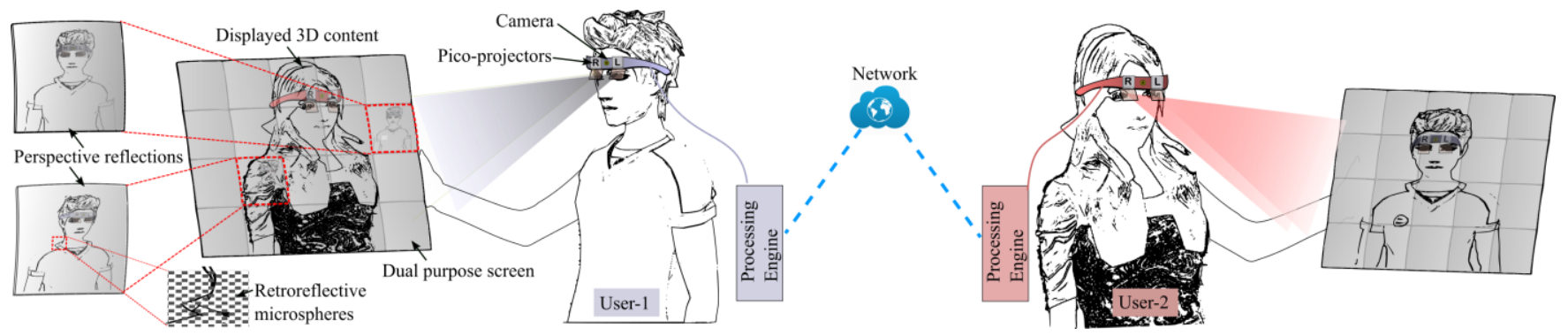

Figure 1: Shows the conceptual illustration of the 3D telepresence architecture using head-worn devices and dual-purpose screens. The stereo 3D content is provided on the top retroreflective layer of the screen using head-worn projectors, while 3D perspective views are recorded using the head-worn camera through the reflections of the mirror array hidden in the screen.

\section{Abstract}

Head-mounted displays and augmented reality headsets are emerging as the future of human-computer interaction. Such devices can display high resolution 3D images and use on-board cameras to capture the surroundings of the user. However, capturing the user who is wearing the device to facilitate $3 \mathrm{D}$ telepresence is not possible with such headsets. Here we propose and demonstrate a new integrated platform to provide mobile 3D telepresence experience using a head-worn device and a dualpurpose passive screen. At the core of this telepresence architecture, we use a portable multi-layered passive screen which facilitates the stereoscopic 3D display using a pair of head-worn projectors and at the same time, captures the multi-perspective views of the user on a head-worn camera through reflections of the screen. The screen contains retroreflective material for stereo image display and an array of convex mirrors for $3 \mathrm{D}$ capture. The $3 \mathrm{D}$ telepresence is demonstrated using an experimental setup where a local-user wearing the developed head-worn device perceives the $3 \mathrm{D}$ images on the dual-purpose screen, while the captured perspective views of user-1 are rendered as stereo viewpoints and showed to the user- 2 on a virtual reality headset.

Keywords: 3D Telepresence, 3D Display, 3D Imaging, Wearable devices.

Index Terms: H.5.1 [Information Interfaces and Presentation]: Multimedia Information Systems-Artificial, augmented, and virtual realities.

\section{INTRODUCTION}

The availability of the display and capturing devices at one place is a primary requirement of any telepresence system. Current wearable $3 \mathrm{D}$ displays are quite advanced but provide limited field-of-view and small screen [2,3]. Such devices also use one or

*email: ssoomro13@ku.edu.tr more cameras and depth sensors on the head to capture the surroundings of the user. However, those cameras cannot capture the user of the headset itself, hence no telepresence applications can be facilitated. On the other hand, multi-perspective 3D imaging requires large and distributed camera installations to capture the user/scene from different angles which limits their implementation for mobile applications $[1,4]$. Also, one of the main concerns of the current $3 \mathrm{D}$ telepresence systems is the physical separation of the display and imaging elements.

In this paper, we present a $3 \mathrm{D}$ telepresence system based on the integrated 3D display and imaging approach using a dual-purpose screen, and head-worn projectors and camera. The system shows the $3 \mathrm{D}$ content on a dual-purpose screen using head-worn projectors and captures the multi-perspective views of the user through the same screen. The system can provide immersive telepresence experience by capturing the user from different viewpoints and displaying the captured information interactively.

\section{System OVerview}

Our telepresence concept is based on a head-worn device and a portable dual-purpose screen. The head-worn device contains a pair of pico-projectors and a high-resolution camera, while the dual-purpose screen has multiple optical layers to facilitate $3 \mathrm{D}$ display and imaging simultaneously. The person wearing the device perceives the stereo-3D content on the screen which is illuminated by the head-worn pico-projectors. On the other hand, the head-worn camera captures the multi-perspective $3 \mathrm{D}$ views of the person through the reflections of the same screen.

The screen consists of two optical layers where top layer has micro-patterned retroreflective microspheres [5]. The retroreflective microspheres reflect the projected light back towards projector to provide the bright content and separate the stereo images for each eye. On the other hand, the bottom layer has an array of convex mirrors to create the $3 \mathrm{D}$ views of the user which are captured by the high-resolution head-worn camera in a single shot. The separation between display and imaging is facilitated by using mutually perpendicular polarization for camera and projectors [6].

The 3D telepresence is envisioned by having two users: user-1 and user-2, each wearing the proposed head-worn device and holding the dual-purpose screen as illustrated in Figure. 1. The 
head-worn camera captures the multiple perspective views of the user-1 in the single shot which are rendered into the set of stereo $3 \mathrm{D}$ pairs. A selected stereo image pair from the available 3D views is transmitted to the user-2 and displayed on his/her screen using head-worn projectors. The selection of stereo views is performed by the user- 2 to perceive the user- 1 from different viewpoints. To provide the bidirectional communication, the $3 \mathrm{D}$ views of user- 2 are also transmitted to the user- 1 in the same way.

\section{Experimental Demonstration and Discussion}

We developed an experimental setup to demonstrate the $3 \mathrm{D}$ telepresence. Our head-worn unit (shown in Figure 2(a)) consisted two off-the-shelf laser scanning pico-projectors where each projector provided 15 lumen lights and $848 \times 480$ pixels resolution. The distance between the exit-pupils of the projector was adjusted to $6.5 \mathrm{~cm}$. A beam-splitter was used between eye and projector to provide high brightness and minimize the crosstalk between the views. For 3D capture, a 24-megapixel photography camera was attached to the head-worn unit.
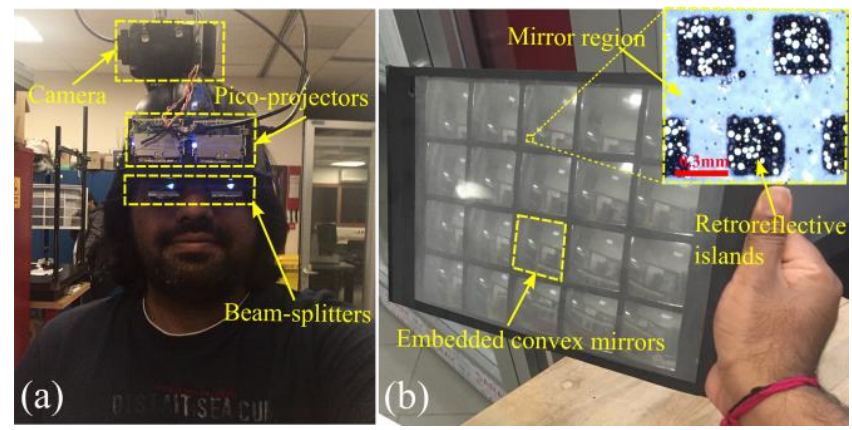

Figure 2: Shows the experimental prototype (a) Head-worn device, and (b) Dual-purpose passive screen.

The dual-purpose screen consisted $5 \times 4$ array of convex mirrors as the bottom layer to provide 20 different perspective views of the user as shown in figure $2(\mathrm{~b})$. The close view of the screen (figure 2(b) inset) shows square shaped islands which are retroreflective microspheres which gap between the islands used by mirrors for imaging. The focal length and size of each mirror was $7 \mathrm{~cm}$ and $5 \mathrm{~cm}$ respectively. $25 \%$ of the total screen area was used for display while remaining $75 \%$ area was utilized for imaging.

Figure 3 shows the experimental demonstration of the 3D telepresence. Our current implementation is limited to the single set of head-worn device and dual-purpose screen as shown in figure 3(a), therefore we used a virtual reality headset (oculus rift DK2) as the display interface for the user-2 as shown in figure 3(b). User-1 with head-worn device and passive screen perceives the $3 \mathrm{D}$ views on the screen (which can be received from the user2). Figure 3(c) shows the left and right views as perceived by the user-1. At the same time, the camera on the head-worn unit captures the $3 \mathrm{D}$ views of the user- 1 and relays them to headset of the user-2 as shown in Figure 3(d). Since there are 20 available views (16 stereo viewpoints), user-2 can hop between the available viewpoints interactively. The brightness of the display was determined as $>150 \mathrm{~cd} / \mathrm{m}^{2}$ per eye, while the crosstalk in stereo mode was $<4 \%$ at the working distance of $70 \mathrm{~cm}$. The capture resolution of $\approx 0.4 \mathrm{MP}$ and $\approx 1 \mathrm{MP}$ per view was achieved by head-worn camera for real-time video streaming and still photos respectively.

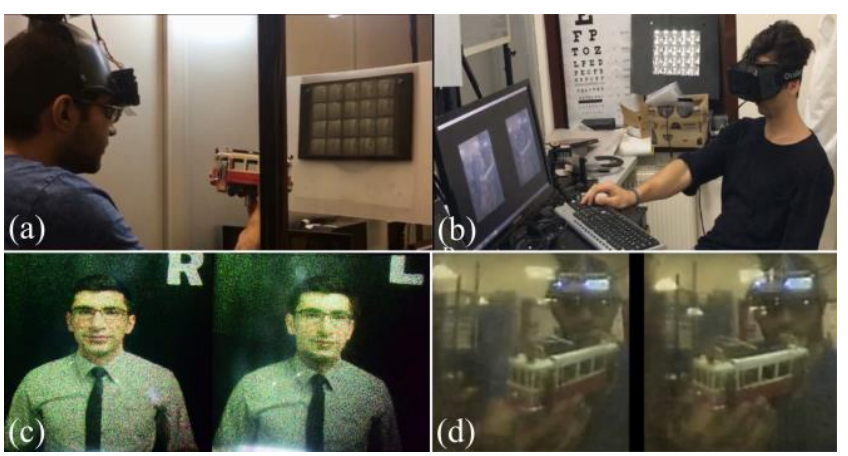

Figure 3: (a) Shows user-1 with developed prototype, (b) shows a user-2 wearing VR headset connected to the user-1, (c) Stereo 3D images projected on the screen and perceived by user-1, and (d) The content perceived by user- 2 on the VR headset.

\section{Conclusion And Future Work}

We proposed a new hardware architecture for 3D telepresence applications using a projection-based head-worn device and a portable dual-purpose passive screen. The screen provides bright stereo 3D imagery illuminated with head-worn projectors and captures the multi-perspective $3 \mathrm{D}$ views of the user/scene simultaneously. The premise of the proposed technique is demonstrated by developing a tabletop setup having a head-worn device and portable screen. The captured 3D views of the user were rendered as stereo pairs and relayed to the remote-end on a virtual reality headset.

The future work includes the development of another identical device and the screen to demonstrate bidirectional 3D telepresence. The quality of captured images can be improved by refining the screen fabrication process or using high quality lenses. The transmission through internet can be included by situating the local and remote users at different places.

\section{ACKNOWLEDGMENTS}

This work is funded by the European Research Council (ERC) under the European Union's Seventh Framework Program (FP7/2007-2013) / ERC advanced grant agreement (340200) and ERC Proof-of-Concept grant no. 755154.

\section{RefERENCES}

[1] B. Javidi et al., "Multidimensional Optical Sensing and Imaging System ( MOSIS ): From Macroscales to Microscales," Proc. IEEE, vol. 105 , no. 5, pp. 850-875, 2017.

[2] K. Kiyokawa, "Head-Mounted Display Technologies for Augmented Reality," in Fundamentals of Wearable Computers and Augmented Reality, Woodrow Barfield, Ed. Tylor and Francis Group, 2016, p. Chapter 4.

[3] B. Kress and T. Starner, "A review of head-mounted displays (HMD) technologies and applications for consumer electronics," in SPIE Proceedings, 2013, vol. 8720, p. 87200A-87200A-13.

[4] M. Martinez-Corral, A. Dorado, J. C. Barreiro, G. Saavedra, and B. Javidi, "Recent Advances in the Capture and Display of Macroscopic and Microscopic 3-D Scenes by Integral Imaging," Proc. IEEE, vol. 105, no. 5, pp. 825-836, 2017.

[5] S. R. Soomro and H. Urey, "Light-efficient augmented reality 3D display using highly transparent retro-reflective screen," Appl. Opt., vol. 56, no. 22, pp. 6108-6113, 2017.

[6] S. R. Soomro and H. Urey, "Integrated 3D Display and Imaging Using Dual-Purpose Passive Screen and Head-Mounted Projectors and Camera," Opt. Express, vol. 26, no. 2, 2018. 